\title{
Utilisation de la carte conceptuelle auprès de formateurs en santé pour l'apprentissage de concepts pédagogiques
}

Caire MARCHAND*, Rémi GAGNAYRE*

\begin{abstract}
Résumé Contexte : Cet article présente une expéri ence pédagogique au cours de laquelle a été introduite la technique des cartes conceptuel les pour l'apprentissage du concept de métacognition, auprès de formateurs de profession nels de santé M éthode: dans un cadre d'autoformation, il a éé proposé aux participants de réaliser trois cartes conceptuelles à partir du concept de métacognition, à 5 mois d'intervalle. Les cartes conceptuelles ont été envoyées, accompagnées de commentaires rédigés par les partici pants concernant leur point de vue sur leur propre travail. Les cartes con ceptuelles ont été analysées en terme de nature et d'organisati on des connaissances, ains que les commentaires les accompagnant. Résultats : D ouze cartes conceptuelles réal isées par 4 partici pants ont été analysées avec les commentai res correspondants. L'évolution des cartes conceptuelles des 4 participants montre une réelle appropriation de ce concept, les connaissances s'enrichissent et s'organisent progressivement (augmentation et précision des concepts et des liens, mise en lien des domaines de connaissances). Si les participants ont éprouvé des difficultés au cours de l'élaboration de la première carte, tous expriment leur satisfaction en ce qui concerne les bénéfices acquis à l'issue de l'expérience. Conclusion : la carte conceptuelle consitue une technique intéressante pour l'apprentissage d'un nouveau concept lors d'activité d'autoformation car elle incite, simultanément à son élaboration, à une réflexion métacognitive.
\end{abstract}

Mots Clés Carte conceptuelle; métacognition ; formation de formateurs en santé; autoformation.

Summary context: This article describes an innovative experience in which concept mapping has been used, with healthcare teachers, to improve their learning of the concept of metacognition. Method: Aspart of self training, learners were asked to draw up three concept maps on the concept of metacognition, at 5 month interval. Each concept map was sent with personal comments from the learner. The nature and the organization of knowledge as well as the learner's comments were analyzed from this concept mapping. Results: Twelve concept maps, drawned by 4 learners, were analyzed with the comments. The evolution of the 4 learners' concept maps shows a real appropriation of the concept. Knowledge is richer and better organized (wider and better defined concepts, linkage of various knowledgefields). While learners en countered some difficulties in the preparation of the first concept mapping, all of them expressed satiffaction regarding benefits obtained at the end of experience. Conclusion: Concept mapping appears to be a very interesting technique for the learning of new concept during self training activities, because it triggers metacognitive thinking during the process.

Key word S concept mapping; metacognition; training of health sciencesteachers, pedagogical training; seff training. Pédagogie M édicale $2004 ; 5$ : 13-23

\footnotetext{
* Laboratoire de pédagogie de la Santé, UPRES EA 3412, Université Paris 13, URR SMBH Léonard de Vinci, 74 rue Marcel Cachin, 93017 Bobigny Cedex, France
}

Correspondance: Caire MARCHAND, Laboratoire de pédagogie de la Santé, UPRES EA 3412, Université Paris 13, UPR SMBH Léonard de Vinci, 74 rue Marcel Cachin, 93017 Bobigny Cedex, France - Tél. : +33 148388898 - Fax : +33 148387619 mailto:c.marchand@smbh.univ-paris13.fr 


\section{Recherche et Perspectives}

\section{Introduction}

Former des formateurs de professionnels de santé consiste à développer, chez eux, des capacités ou des potentialités de compétences. Ces capacités transférées et mises en œuvre par les participants dans leur pratique professionnelle révèlent alors les compétences réellement acquises. Ces transferts nécessitent que soient dével oppées par les participants, pendant la formation, des capacités denature métacognitive ${ }^{1,2}$. La métacognition est définie comme l'analyse de son propre fonctionnement intellectuel, une capacité d'auto-analyse des systèmes de traitement de l'information que tout individu met en œuvre pour apprendre, se souvenir, résoudre des problèmes ou conduire une activitéz. Ainsi, la métacognition aurait pour but d'élargir le champ de conscience de l'apprenant et donc sa capacitéà réutiliser ce qu'il sait dans des contextes différents.

Le terme demétacognition restepour beaucoup deformateurs en santé une notion peu connue, un concept nouveau à intégrer puis à opérationnal iser pour permettre son développement et son transfert auprès des étudiants ou des professionnels desanté en formation continue.

L'introduction d'un nouveau concept nécessite un réel apprentissage. Selon la psychologie cognitive, ce dernier se réalise lorsque l'apprenant met en lien l'information nouvelle avec son réseau de connaissances préal ables, transformant sa structure cognitive ou I'organisation de ses connaissances'. En lien avec ce dernier aspect, plusieurs auteurs ont souligné la relation qui existe entre l'organisation des connaissances et le niveau d'expertise d'une personnet. Autrement dit, plus les connai ssances sont organisées, reliées entre elles sous forme de réseau, meilleures sont les capacités à résoudre des problèmes ou à prendre des décisions. Pour favoriser l'organisation des connaissances chez les apprenants, d'autres études ont montré l'intérêt d'utiliser la technique des cartes conceptuelles, 6,7 . Une carte conceptuelle est une représentation graphique, hié rarchisée, d'un ensemble de concepts et de liens de sens qu'un individu établit entre les différents concepts ${ }^{8}$. Elles ont été initialement conçues pour améliorer l'apprentissage en favorisant la construction et l'organisation des connaissances, rendant l'apprentissage plus signifiant, plus profond, comparativement à un apprentissage «par cœeur » 6,7. Au-delà d'une aide à l'apprentissage, les cartes conceptuelles peuvent aussi être utilisées comme outil de planification d'une formation', ou encore comme instrument d'évaluation des connaissances ${ }^{10}$. Enfin, les cartes conceptuelles peuvent être élaborées avec toutes sortes d'appre- nants puisque nous les avons particulièrement utilisées pour explorer les connaissances antérieures de patients atteints de maladies chroniques (diabétiques, obèses) ainsi quepour apprécier l'évolution deleurs connaissancesà l'issue et à distance d'une période d'éducation thérapeutique $^{11,12}$. Cet article présente une innovation pédagogique au cours de laquelle des cartes conceptuelles ont été utilisées pour favoriser l'apprentissage du concept «métacognition » par des formateurs en santé au cours d'une formation pédagogique Par ailleurs, l'innovation comporte une dimension évaluative sur la valeur pédagogique de cette activité. C ette innovation s'inscrit donc dans une démarche d'évaluation de programme de formation ${ }^{13}$.

\section{Description del'expérience}

\section{Contexte et principes théoriques}

L'expérience a été menée auprès d'un groupe d'une vingtaine de formateurs de professionnels de la santé (formateurs infirmiers, sages-femmes, médecins, dentistes, etc.), inscrits pour suivre un diplôme universitaire de pédagogie de la santé. C etteformation, organiséeen alternance, comprend une dizaine de modules de 3 à 4 jours. Elle a pour but de faire acquérir aux participants des compétences méthodologiques de formation leur permettant d'améliorer leurs pratiques professionnelles de formateur en santé. D ansuneformation pédagogique, l'apprentissageà l'autoformation est un des objectifs fondamentaux. Pour initier et développer des compétences appropriées d'autoforma tion, nous proposons une activitéqui consisteà utiliser les cartes conceptuelles pour l'apprentissage de concepts pédagogiques. Cette production de cartes conceptuelles, individuelle, personnalisée, est adressée à un tuteur dont la tâche princi pale consiste à susciter la motivation du participant et à lui proposer des ressources pédagogiques (réfé rences bibliographiques, conseils de lecture, de formation, personnes ressources, etc.). La validation du contenu des cartes conceptuelles est réal isée par le participant lui-même au fur et à mesure de sa formation, à l'occasion de ses lectures, des rencontres avec les enseignants et participants et des travaux écrits qu'il doit réaliser. Cette position tutorale nous semble ainsi plus cohérente avec les principes d'une autoformation ${ }^{14}$ qui sappuie sur la motivation intrinsèque du participant.

\section{Introduction de l'activité}

A l'issue du premier séminaire de 4 jours de formation, nous avons introduit la technique des cartes conceptuelles. 
La méthodologie d'élaboration d'une carte conceptuelle, telle qu'elle a été décrite par N ovak et Gowin ${ }^{8}$, a été pré sentée aux participants : à partir d'un concept central, fai re la liste des concepts importants, les organiser et les reporter sous la forme d'une carte conceptuelle (les concepts symbolisés dans des bulles, les liens signalés par des flèches et explicités). D es exemples de cartes conceptuelles ont été présentés. N ous avons proposé à l'ensemble des participants de réaliser individuellement une carte conceptuelleà partir du concept «métacognition ». Le choix de ce concept repose sur les raisons suivantes: comme nous l'avons souligné plus haut, le développement de la métacognition est une condition de transfert des compétences; les repères pratiques issus de la recherche montrent qu'il sagit d'un concept d'appropriation difficile d'où notre volonté d'expérimenter de nouvelles stratégies d'enseignement et apprentissage (la carte conceptuelle) ; et enfin, la découverte de ce concept par les participants eux-mêmes renvoie à une réflexion métacognitive formative.

Un temps de discussion a suivi cet exercice afin de laisser s'exprimer les impressions et difficultés rencontrées par les participants. Chaque participant a conservé sa carte conceptuelle.

Il a ensuite été proposé à ceux qui le désiraient (sans aucune obligation étant donnéqu'il serait contre productif « d'imposer » une autoformation), de participer à une expérience pédagogique. Les consignes suivantes ont été données:

- conserver cette première carte conceptuelle (CC1), la dater ;

- réal iser une deuxième carte conceptuelle (CC2) puis une troisième carte $(\mathrm{C} C 3)$, respectivement 5 mois puis 10 mois après la première carte. Sur une feuille accompagnant les cartes préciser les éléments suivants: quels sont les changements que vous constatez dans votre carte? A quoi ces changements peuvent-ils être attribués (modules de formation, échanges avec des collègues et des experts, lectures, etc.) ? Q uels aspects vous semblent encore flous, imprécis (nécessitant des clarifications, des compléments d'information, vous posant des questions) ? U ne partie était réservéeà des commentaires personnels.

- Une rétro-information a été envoyée à chaque participant après la réception des deux premières cartes (la première carte a été élaborée en septembre, conservée par le participant et envoyée en même temps que la deuxième réal isée en janvier) et avant l'élaboration de la troisième réal isée environ 5 mois après. Elle avait pour objectif principal de les rassurer sur leur production, de les inviter à poursuivrel'expérience, de répondre aux éventuelles questions méthodologiques posées par les participants.

\section{Analyse des cartes conceptuelles}

N ous avons réalisé une analyse qualitative concernant la nature et l'organisation des connaissances pour apprécier les évolutions entre les trois cartes. Les critères d'analyse choisis sappuient sur la théorie d'Ausube ${ }^{15}$ concernant la construction de la connaissance, ainsi que sur les critères d'analysequalitative proposés par Kinchin et ses collabora teurs ${ }^{16}$. Ces mêmes critères nous ont permisd'élaborer une grille d'analyse pour des travaux portant sur I'utilisation des cartes conceptuelles auprès de patients atteints de mal adie chronique dans le cadre de leur éducation ${ }^{11}$. C ette anal yse n'avait pas pour objectif de comparer les cartes conceptuelles des participants mais de repérer chez chacun d'eux des indicateurs de l'évolution de l'apprentissage.

Leséléments suivants ont été recherchés:

- Le nombre et la nature des domaines de connaissances identifiés. Selon Ausubel, tout nouveau concept vient sincorporer à un concept plus large dit de « superordination $»^{5}$. D ans notre étude, un domaine de connaissance correspond à un ensemble de connai ssances élaboréautour d'un concept relativement global. L'ensemble des connaissances autour de ce concept définit un domain $\mathrm{e}^{11,12}$ (par exemple: domaines de la définition ou des buts du concept).

- Le nombre et la nature des concepts pouvant témoigner d'un enrichissement des connaissances (nombre de concepts identiques, nombre de concepts nouveaux).

- Le nombre de liens reliant les différents domaines et caractérisant la nature plus ou moins structurée des connaissances.

- Le type de structure révélée par la carte. Trois structures ont été décrites par Kinchin et ses collaborateurs ${ }^{16}$ : - une structure en étoile dans laquelle tout concept est uniquement relié au concept central. D ans ce cas, il n'existe qu'une seule possibilité d'accès aux connaissances ce qui limite l'apprentissage. L'ajout d'un nouveau concept par l'apprenant ne modifie pas fondamentalement l'organisation de ses connaissances ;

- une structuration en chaîne avec laquelle il reste difficile d'ajouter un nouveau concept sans perturber I'ensemble de la chaîne. Ce type d'organisation peut donc faire obstacle à l'intégration de nouvelles connaissances;

- et enfin, I'organisation des connaissances en réseau qui témoigne de nombreux liens entre des domaines de 


\section{Recherche et Perspectives}

connaissances différents. C ette organisation semble la plus complexe, la plus évolutive et opérationnelle car elle propose de nombreuses entrées pour l'intégration de nouveaux concepts.

\section{Résultats et analyse des cartes \\ Les sujets de l'expérience}

Q uatre professionnels ont spontanément participé à l'étude: un infirmier, enseignant dans un Institut de Soins Infirmiers, une infirmière anesthésiste en seignante dans un C entre d'Enseignement de Soins d'U rgence (CESU ), une infirmière menant des actions d'éducation thérapeutique auprès de patients atteints de maladies cardiaques, une sage-femme indépendante menant des actions de formation de préparation à l'accouchement. Les participants étaient tous en première année du diplôme et ont suivi, au cours de cette année, 4 modules de formation sur les thèmes suivants:

- «Initiation à la pédagogie de la santé », ayant pour objectif essentiel de permettre aux participants d'analyser leur pratique pédagogique en regard de courants et méthodes pédagogiques.

- «Techniqued'animation et conduite de groupe», dont I'objectif est l'acquisition des principes de la dynamique de groupe et de techniques pédagogiques actives.

- «Planification de programme de formation », développant chez les participants leur capacité à concevoir l'architecture pédagogique de cursus de formation.

- «Stratégies d'enseignement », dont l'objectif est de permettre de concevoir une intervention de formation et de la gérer dans une perspective de psychologie cognitive.

\section{Analyse des cartes}

12 cartes conceptuelles ont été réalisées et analysées. A titre d'illustration, les figures 1,2 et 3 présentent les cartes conceptuelles réalisées par un des sujets (S4).

Le tableau 1 propose une synthèse des éléments d'analyse obtenus à partir des 3 cartes conceptuelles réalisées par 4 sujets. II reprend les principales évolutions observées entre la première carte (CC1) et la deuxième carte (CC2), puis les évolutions observées dans la troisième carte (CC 3).

En CC 1, deux cartes conceptuelles sur 4 révèlent une méconnai ssance totale du concept lui-même (cartes des sujets S2 et S3). Ces deux cartes subissent une transformation radicale en $\mathrm{CC} 2$ : très peu de concepts exprimés en C C 1 sont conservés et de nouveaux concepts et domaines de connaissances apparaissent. Les deux autres sujets ( $\mathrm{S} 1$ et $\mathrm{S} 4$ ) enrichissent leur première carte en précisant certains concepts (ajout de concepts et de liens), en substituant un concept par un autre concept plus précis, ou encore en ajoutant de nouveaux liens entre des concepts existants. Les quatre sujets réalisent une troisième carte conceptuelle bien plus riche que les deux premières : en richissement des domaines de connaissances existants (augmentation du nombre de concepts et de liens), apparition de nouveaux domaines de connaissances (en lien par exemple avec les habiletés métacognitives et le transfert). La troisième carte des sujets S2 et S3 montre une nette appropriation de la notion de métacognition (précision en termes de définition et de buts). Pour trois sujets, la structure générale des deux premières cartes produites (CC1 et CC2) est mixte : structuration en étoileet en chaîne. Un seul sujet (S3) élabore, dès le début, des cartes où les connaissances sont présentées en réseau. Les troisièmes cartes conceptuelles (CC 3) laissent apparaitre chez les 4 sujets des prémices d'organisation en réseau, certains domaines de connaissances étant reliés entre eux.

\section{Synthèse des commentaires}

Le tableau 2 reprend les principaux commentaires exprimés par les participants de l'innovation aux différentes phases de l'étude : les changements constatés, les sources d'information à l'origine de ces changements, les besoins de clarification des sujets et leurs commentaires personnels.

Pour les 4 sujets de l'étude, ce sont les modules de formation et certaines lectures qui leur ont permis d'améliorer leurs cartes conceptuelles. La rétro information sur les deux premières cartes ainsi qu'une réflexion personnelle sont des éléments qui ont aidé le sujet S2 à améliorer la réalisation de ses cartes. Les interrogations principales qui subsistent à l'issue de la réalisation de la troisième carte concernent l'aspect opératoire de la métacognition : quelles techniques permettent de déve lopper la métacognition (cité par 3 sujets S1, S3 et S4) ? Les participants s'expriment sur leurs difficultés au moment de la réalisation de la carte : cette activité trouve un intérêt auprès d'un sujet (S3) uniquement à la troisième étape (CC 3). En revanche, un autre sujet (S1) souligne l'ampleur des connaissances acquises, lui posant un problème d'organisation spatiale pour une représentation sous forme de carte conceptuelle. 
Utilisation de la carte conceptuelle auprès de formateurs...

\section{Figure 1 : Première carte conceptuelle élaborée par le participant S4}

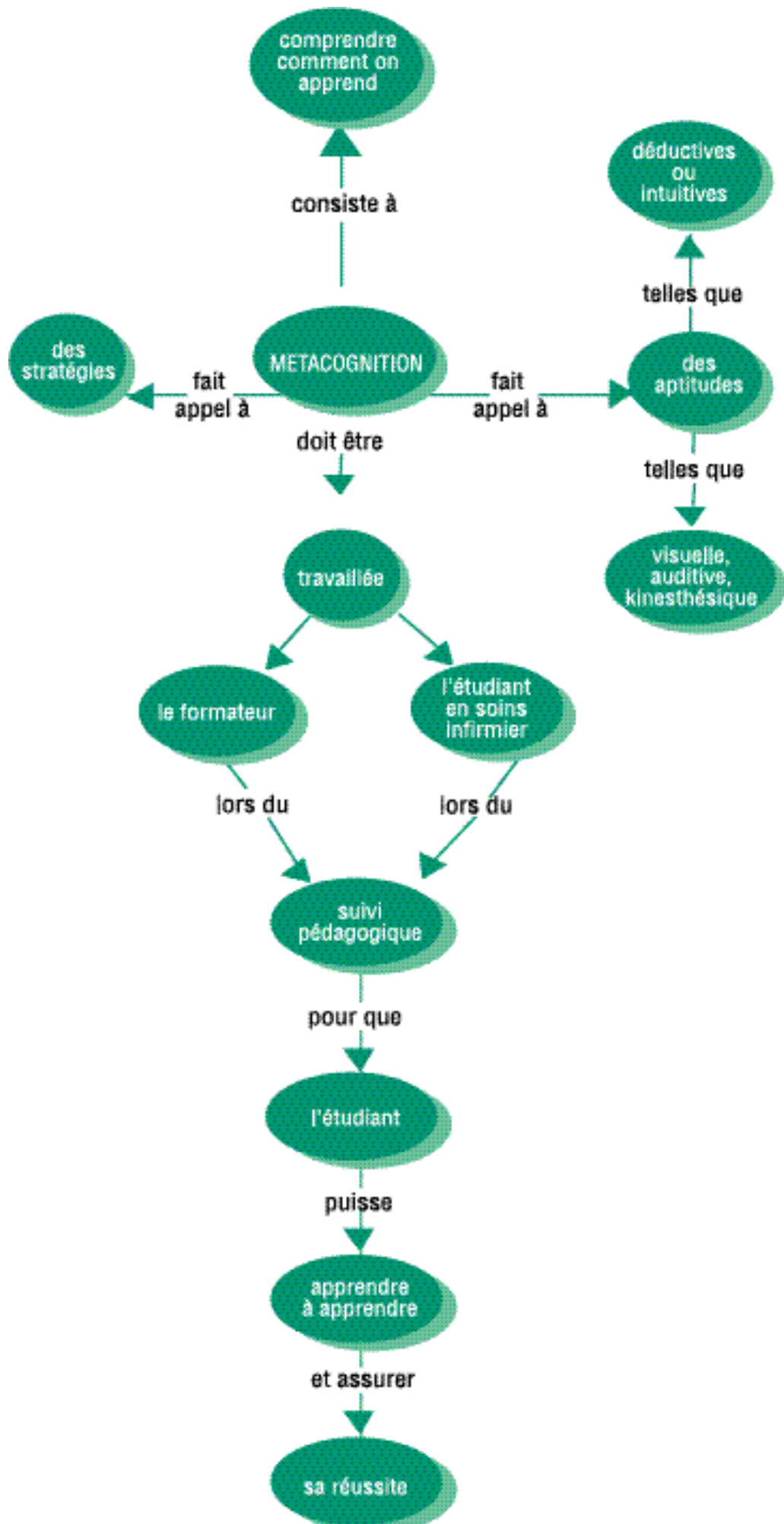




\section{Recherche et Perspectives}

Figure 2 : D euxième carte conceptuelle réalisée par le participant S4

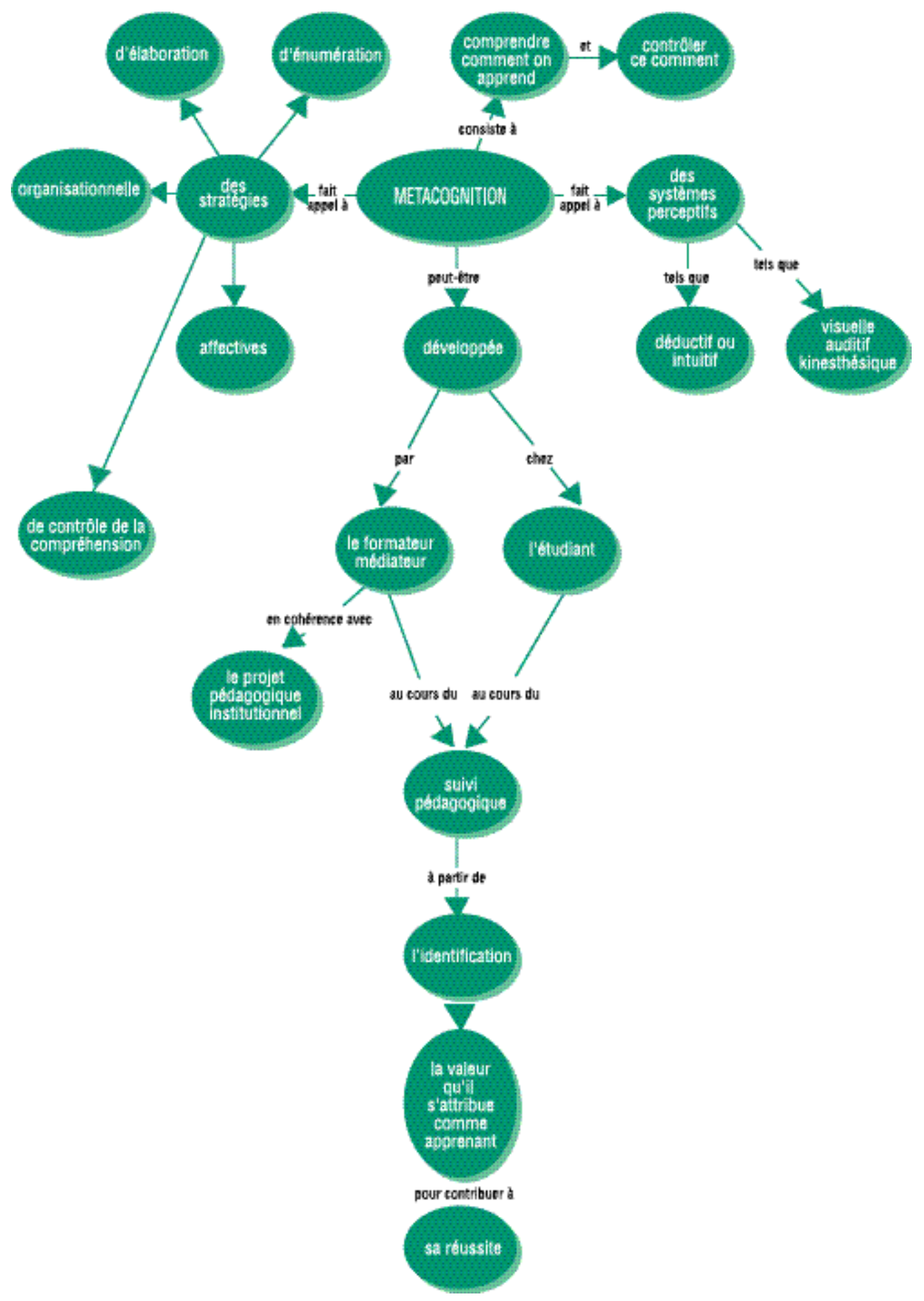


Utilisation de la carte conceptuelle auprès de formateurs...

Figure 3 : Troisième carte conceptuelle réalisée par le participant S4

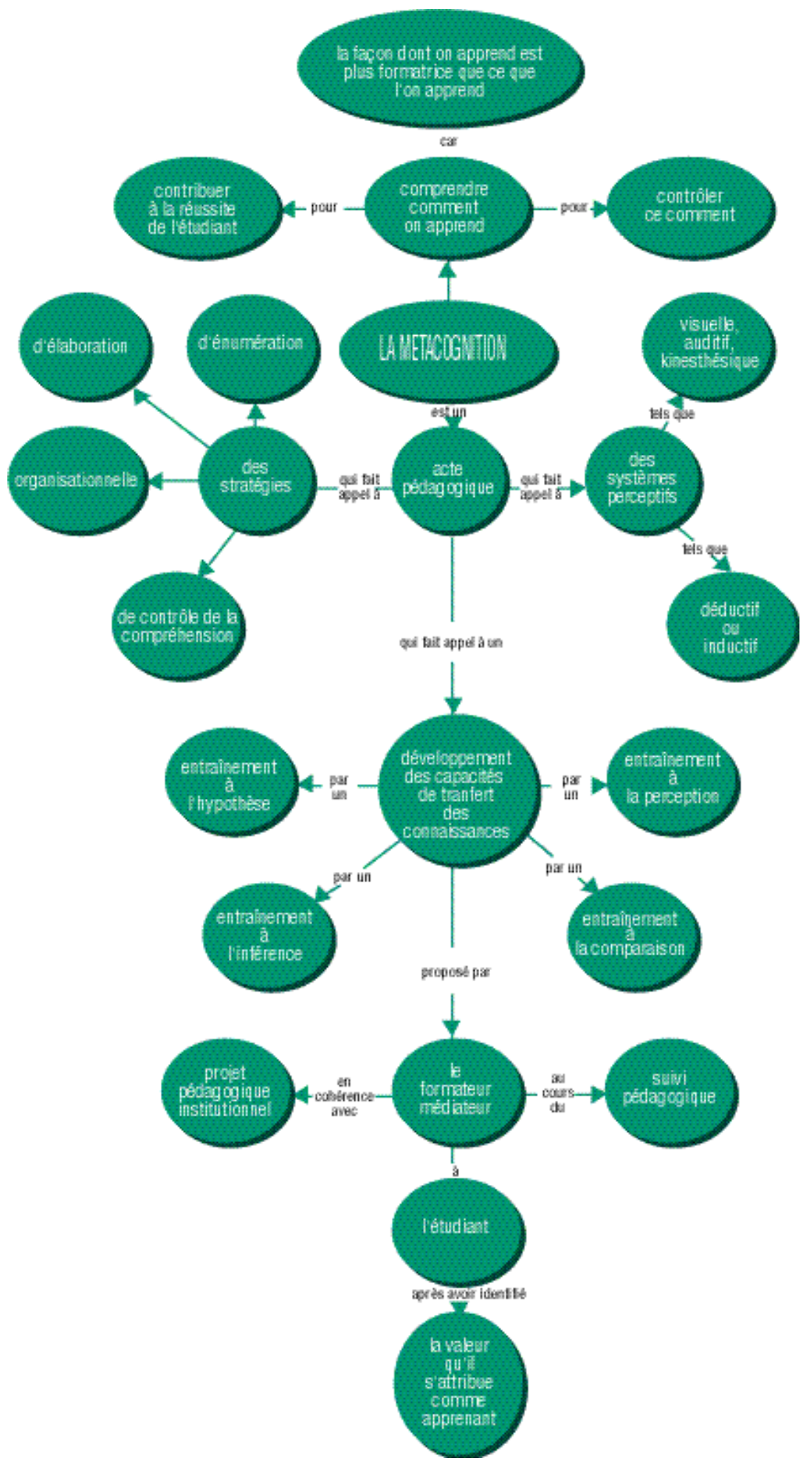




\section{Recherche et Perspectives}

\begin{tabular}{|c|c|c|c|c|}
\hline \multicolumn{5}{|c|}{ Tableau 1 : Synthèse de l'analyse des cartes conceptuelles } \\
\hline Participants & S1* & S2 & S3 & S4 \\
\hline $\begin{array}{l}\text { Evolutions observées } \\
\text { entre la première } \\
\text { (CC 1) et la deuxième } \\
\text { cartes (CC 2) }\end{array}$ & $\begin{array}{l}\text { - Précision destermes } \\
\text { et connaissances } \\
\text { nouvelles sur les } \\
\text { références théoriques } \\
\text { - Modification de la } \\
\text { définition de la } \\
\text { métacognition } \\
\text { - Clarification des liens } \\
\text { entre méta cognition } \\
\text { et pédagogie } \\
\text { - Réorganisation géné } \\
\text { rale dela carte: } \\
\text { deux nouvelles mises } \\
\text { en lien } \\
\text { - Trois puis } 5 \text { domaines } \\
\text { de connaissances: } \\
\text { «définition, rôle dans } \\
\text { l'apprentissage, } \\
\text { qu'est-ce que cela fait } \\
\text { intervenir, acteurs, } \\
\text { origine» } \\
\text { - Structuration mixte: } \\
\text { étoile et châne }\end{array}$ & $\begin{array}{l}\text { - Transformation totale } \\
\text { de la carte: unique- } \\
\text { ment } 4 \text { concepts } \\
\text { identiques, } \\
9 \text { nouveaux liens } \\
\text { - Concept de méta } \\
\text { cognition non acquis } \\
\text { - } 4 \text { domaines de } \\
\text { connaissancesen } \\
\text { CC } 1 \text {, aucun en CC2 : } \\
\text { - structuration } \\
\text { en étoile }\end{array}$ & $\begin{array}{l}\text { - Transformation totale } \\
\text { de la carte: unique } \\
\text { ment deux concepts } \\
\text { sont retrouvés en } \\
\text { CC } 1 \text { et CC 2, } \\
10 \text { nouveaux } \\
\text { concepts en CC2 } 2 \\
\text { et } 2 \text { nouveaux liens } \\
\text { - } 2 \text { puis } 4 \text { domaines de } \\
\text { connaissances: } \\
\text { «étudiant, ensej- } \\
\text { gnant, type de } \\
\text { connaissances, } \\
\text { démarche d'appren- } \\
\text { tissage » } \\
\text { - ensemble des } \\
\text { domaines reliés } \\
\text { entre eux } \\
\text { - structuration en } \\
\text { réseau }\end{array}$ & $\begin{array}{l}\text { - Enrichissement dela } \\
\text { carte par complé } \\
\text { ments apportés dans } \\
\text { certains domaines de } \\
\text { connaissances } \\
\text { (11 nouveaux } \\
\text { conceptset } 7 \text { nou- } \\
\text { veaux liens) } \\
\text { - Transformation de } \\
\text { certains concepts } \\
\text { (ex: formateur } \\
\text { devient formateur- } \\
\text { médiateur) } \\
\text { - } 3 \text { domaines de } \\
\text { connai ssances sont } \\
\text { identifiés: «finali- } \\
\text { tés/buts, défintition, } \\
\text { acteurs concernés» } \\
\text { - structuration mixte: } \\
\text { étoile et châne }\end{array}$ \\
\hline $\begin{array}{l}\text { Evolutions observées } \\
\text { entre la deuxième } \\
\text { carte (C C 2) et la } \\
\text { troisième carte (C C 3) }\end{array}$ & $\begin{array}{l}\text { - Enrichissement de la } \\
\text { carte (concepts et } \\
\text { liens) } \\
\text { - Miseen lien de la } \\
\text { définition avec } \\
\text { d'autres notions: } \\
3 \text { nouveaux } \\
\text { domaines: habiletés } \\
\text { métacognitives, } \\
\text { connaissances méta } \\
\text { cognitives, techniques } \\
\text { - Développement du } \\
\text { domainede connais- } \\
\text { sances concernant les } \\
\text { techniquespermet- } \\
\text { tant de développer la } \\
\text { métacognition } \\
\text { - Sélection des } \\
\text { concepts majeurs } \\
\text { pour la compréhen- } \\
\text { sion du terme } \\
\text { (disparition de cer- } \\
\text { tains concepts) } \\
\text { - Structuration en } \\
\text { réseau }\end{array}$ & $\begin{array}{l}\text { - Précision du concept } \\
\text { de méta cognition et } \\
\text { des liens reliant les } \\
\text { concepts entre eux } \\
\text { (8 nouveaux liens et } \\
5 \text { nouveaux concepts) } \\
\text { - Seul le concept de } \\
\text { réflexion est retrouvé } \\
\text { dans les } 3 \text { cartes } \\
\text { conceptuelles } \\
\text { - } 2 \text { domaines de } \\
\text { connaissances: } \\
\text { «connaissances, } \\
\text { cogitation » } \\
\text { - Structuration en } \\
\text { réseau }\end{array}$ & $\begin{array}{l}\text { - Enrichissement dela } \\
\text { carte (+15 nouveaux } \\
\text { concepts et +17 nou- } \\
\text { veaux liens) } \\
\text { - Apport de précision : } \\
\text { définition et buts } \\
\text { - } 4 \text { domaines de } \\
\text { connaissances: «type } \\
\text { de connaissance, } \\
\text { définition, buts, } \\
\text { en quoi cela consiste» } \\
\text { - Structuration en } \\
\text { réseau }\end{array}$ & $\begin{array}{l}\text { - Enrichissement des } \\
\text { domaines de } \\
\text { connaissances } \\
\text { - N ouveau domaine de } \\
\text { connaissances: «rôle } \\
\text { dans le transfert des } \\
\text { connaissances » } \\
\text { - M ême structure géné } \\
\text { rale en réseau : } \\
\text { organisation des } \\
\text { connaissances autour } \\
\text { de la notion d'acte } \\
\text { pédagogique }\end{array}$ \\
\hline $\begin{array}{l}\text { *N ote: Pour } \mathrm{S1} \text {, il a été } r \\
\text { symbol isé les concepts dans }\end{array}$ & s des bulles, et ayant subst & titué le concept avec des ph & hrases plus ou moinslongu & $\begin{array}{l}\text { cipant n'ayant pas } \\
\text { ues. }\end{array}$ \\
\hline
\end{tabular}


Tableau 2 : Synthèse des commentaires personnels accompagnant les cartes conceptuelles

\begin{tabular}{|c|c|c|c|c|}
\hline Participants & S1* & S2 & S3 & S4 \\
\hline $\begin{array}{l}\text { Principaux } \\
\text { changements } \\
\text { constatés }\end{array}$ & $\begin{array}{l}\text {-Entre CC 1 et CC 2 : } \\
\text { «la définition du concept de } \\
\text { métacognition sest affi née, } \\
\text { l'orignedu concept sest éclair- } \\
\text { cie et les liens entre la pédago- } \\
\text { gie et la métacogniti on me } \\
\text { semblent plus clairs» } \\
\text { - CC3: «le stade de la défini- } \\
\text { tion fut dépassé, permettant } \\
\text { d'aborder les connai ssances et } \\
\text { leshabi letés méta cognitives, et } \\
\text { les techniques qui permettent } \\
\text { de développer la métacogni- } \\
\text { tion» }\end{array}$ & $\begin{array}{l}\text { - « les changements les plus } \\
\text { sgnificatifs sont sûrement le } \\
\text { fait que cette image se rap- } \\
\text { proche un peu plus de moi » } \\
\text { - «j'ai mis la di rection des } \\
\text { liens, inexistants en CC1 et } \\
\text { CC2 }\end{array}$ & $\begin{array}{l}\text {-A propos de CC3: } \\
\text { «cettecarteest loin d'êrecom- } \\
\text { plète mais il me semble qu'elle } \\
\text { est mieux construite que les } \\
\text { deux autres» }\end{array}$ & $\begin{array}{l}\text { - Entre CC1 et C C2 : } \\
\text { «... enrichissement du concept } \\
\text { tel quejeleperçois, ou rempla- } \\
\text { cement d'un terme par un } \\
\text { autre plus précis, ou plus } \\
\text { appropriéà mon sens... » }\end{array}$ \\
\hline $\begin{array}{l}\text { O uvrages } \\
\text { ou sources } \\
\text { d'information }\end{array}$ & $\begin{array}{l}\text { - Participation au } 1^{\text {er }} \\
\text { (Initiation à la pédagogie de } \\
\text { la santé) puis au 4e séminaire } \\
\text { du DU (stratégie d'enseigne } \\
\text { ment) et réali sation du } \\
\text { mémoire d'application } \\
\text { - Premier chapitre du guide } \\
\text { pédagogique, } \\
\text { (JJ. Guilbert) } \\
\text { - Les courants pédagogiques, } \\
\text { (J. Beauté) } \\
\text { - Pour un enseignement straté } \\
\text { gique, (J. Tardif) }\end{array}$ & $\begin{array}{l}\text { - Réflexion personnelle } \\
\text { - C ommentaires reçus à la } \\
\text { suite de la réalisation de la } \\
\text { deuxième carte } \\
\text { - 4e séminaire de D U sur les } \\
\text { stratégies d'enseignement } \\
\text { - « auto- obser vation » dans } \\
\text { mon travail }\end{array}$ & $\begin{array}{l}\text { - Premier séminaire du DU: } \\
\text { initiation à la pédagogie de la } \\
\text { santé } \\
\text { - D eslectures (sans précision) }\end{array}$ & $\begin{array}{l}\text { - Les courants pédagogiques } \\
\text { (J. Beauté) } \\
\text {-Théories contemporaines de } \\
\text { l'éducation, (Y. Bertrand) } \\
\text { - Eduquer et former, (JVC. } \\
\text { Ruano Borbalan) } \\
\text { - L'apprentissage de l'abstrac- } \\
\text { tion, (BM. Barth) }\end{array}$ \\
\hline $\begin{array}{l}\text { D omaines de } \\
\text { connaissances } \\
\text { à clarifier } \\
\text { ou à compléter }\end{array}$ & $\begin{array}{l}\text { - Les modalités d'applica- } \\
\text { tion de la métacognition } \\
\text { - Les stratégies decontrôle } \\
\text { et de régulation, d'éva- } \\
\text { luation et d'auto- } \\
\text { évaluation }\end{array}$ & $\begin{array}{l}\text { - Clarification de la } \\
\text { notion demétacognition }\end{array}$ & $\begin{array}{l}\text { - M étacognition : terme } \\
\text { inconnu, tentative de } \\
\text { rattacher la notion avec } \\
\text { la notion plusconnue de } \\
\text { stratégie d'enseignement }\end{array}$ & $\begin{array}{l}\text { - S'interroge sur les possi- } \\
\text { bilités de développer la } \\
\text { métacognition autre- } \\
\text { ment qu'en guidance au } \\
\text { cours du suivi pédago- } \\
\text { gique }\end{array}$ \\
\hline
\end{tabular}
$\begin{array}{ll}\text { Commentaires } & \cdot \text { Sentiment de } n^{\prime} \text { avoir } \\ \text { personnels } & \text { aucune connaissance au }\end{array}$ début, puis l'élaboration de la carte montrant une certaine organisation a été rassurante

- « difficile d'élaborer une

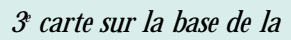
$2^{e}$ qui, à ce stade de ma formation, me semble aborder de nombreux aspects de la métacognition »
- Le flou du projet professionnel se reflète dans le flou de la carte

- Estime que CC2 est plus structurée que $C \mathrm{C} 1$, les termes moins généraux, plus en lien avec la métacognition

- Engage simultanément une réflexion méta cognitive: "j'ai l'impression que le plan d'action de mon processus métacognitif est encore flou... je continuerai à m'introspecter sur mes propres processus méta-cognitifs »
- Exercice réalisé sans plaisir au début, mais commence a entrevoir l'intérêt des cartes conceptuelles à la troisième

- S'interroge sur le rôle de I'enseignant (formateur) pour favoriser chez l'appre nant ses processus d'apprentissage

- Intérêt de se centrer sur l'apprenant et moins sur le formateur
- Interrogation sur les difficultésàmettre en placeune pédagogie de la métacognition : « rapport coût/béné fice, obstacle institutionne, effet néfaste de la métacognition (pouvoir du formateur) 》

- Est-il possible de faire de la métacognition sans le savoir?

- Souhaite faire un lien avec la pédagogie réflexive 


\section{Recherche et Perspectives}

\section{Discussion}

L'analyse des 3 cartes conceptuelles pour chacun des 4 participants montre une netteévolution de la compréhension du terme de métacognition. Ce sont les lectures, puis les modules de formation qui semblent avoir eu le plus d'effets sur l'intégration de ce nouveau concept. Certains ouvrages ont certainement contribué à clarifier les connaissances de deux des participants (f. tableau 2 - S1 et S4). Par ailleurs, le module de formation intitulé : «Stratégies d'enseignement » leur a permis d'aborder plus précisément la notion de métacognition dans ses diffé rentes dimensions (connai ssances et compétences métacognitives, stratégies d'enseignement permettant de développer la métacognition). Les enseignements de deuxième et troisième année devraient les aider à surmonter leurs difficultés pour la mise en œuvre de techniques permettant de développer chez les apprenants leur méacognition. D es modules sur le tutorat et l'évaluation, par exemple, sont planifiés à cet effet.

D eux des sujets de l'innovation expriment leur intérêt nouveau vis-à-vis de la technique des cartes conceptuelles: I'un d'entre eux (S3), réticent au départ, déclare entrevoir progressivement l'intérêt de réali ser ce genre d'exercice (sans autre précision), alors qu'un autre (S1) souligne le pouvoir de la carte conceptuelle à révéler et faire prendre conscience à son auteur du bagage de connaissances préalables qu'il possède et des apprentissages qu'il réalise. Cet effet d'ordre affectif a éé montré lors d'une étude relatant I'utilisation de cartes conceptuelles auprès d'enfants ${ }^{17}$. Ces derniers ont exprimé le plaisir qu'ils ressentaient à voir leurs progrès dans l'organisation et la représentation de leur pensée. N ous avons pu vérifier cet effet dans une étude menée auprès de patients diabétiques qui ont manifesté leur surprise de voir se dessiner devant eux, sous la forme d'une carte conceptuelle, une grande partie de leurs connaissances sur leur maladie ${ }^{11}$. En termes d'effets à plus long terme de cette expérience, il est intéressant de noter qu'un des sujets (S1) a pris I'habitude de synthétiser ses notes de lectures sous la forme de cartes conceptuelles. D eux difficultés peuvent expliquer les résultats constatés lors de la réal isation de la première carte. La première réside dans la découverte d'une nouvelle technique, « la carte conceptuelle». En effet aucun des participantsn'avait entendu parler de cette technique auparavant. $0 \mathrm{r}$, les auteurs s'accordent à dire que pour réaliser une carte conceptuelle de qualité, un véritable apprentissage reste nécessaires. Ce temps d'apprentissage s'est résumé dans notre étude à une simple présentation de la technique.
Cela peut expliquer, d'une part le faible nombre de personnes à avoir accepté de participer à l'expérience faute d'un entraînement conséquent, et d'autrepart, la difficulté qu'ont pu avoir les sujets de l'expérience à représenter immédiatement leurs connaissances sous forme de réseau, ce type d'organisation n'apparaissant réellement qu'au bout de la troisième carte chez trois participants. L'autre difficulté concernele choix du concept qui, pour deux des sujets, était complètement inconnu et semble avoir repré senté un obstacle à l'élaboration de la carte. Cependant, l'expérience montre qu'il est possible de débuter un travail de construction de connaissances à partir d'une notion peu connue, chacun reliant dans un premier temps le concept nouveau à un domaine de connaissances qui lui est plus familier.

Au-delà de la découverte d'une nouvelle technique d'apprentissage, transférable dans la pratique des professionnels en formation, il nous a semblé particulièrement inté ressant de susciter chez les participants de cette étude une véritable réflexion métacognitive Le travail sur le concept de métacognition, les commentaires accompagnant les cartes conceptuelles, le tutorat à distance, et enfin l'élaboration même des cartes conceptuelles sont les ééments qui ont permis de développer chez les participants leur métacognition. Le sujet S2 en est le témoin, ses commentaires personnels portant essentiellement sur sa réflexion méta cognitive (cf. tableau 2). D ans une démarche d'évaluation de programme, l'utilisation de la technique des cartes conceptuel les nécessiterait de consacrer davantage de temps à l'apprentissage de la technique pour permettre à l'ensemble des participants à la formation d'adhérer à l'activité proposée. Un ne autre conséquence serait d'opérer des liens plus étroits et plus explicites entre les lectures des participants et les contenus des modules de formation.

\section{Conclusion}

Cette expérience montre qu'il est intéressant d'utiliser la carte conceptuelle comme activité d'autoformation au cours d'une formation pédagogique. Cet intérêt a été ressenti par les quatre participants puisqu'ils ont participé à l'expérience volontairement et jusqu'à la fin. Certains d'entre eux utilisent maintenant la carte conceptuelle à d'autres fins (notes de lecture, planification d'enseignement, etc.). L'élaboration d'une carte conceptuelle aide à l'apprentissage de concepts abstraits, permet de prendre conscience de ce que l'on sait et oblige à organiser ses connaissances, ce qui constitue un indicateur d'un 
apprentissage signifiant. Au-delà d'un véritable travail sur la clarification et l'organisation des connaissances, il apparaît que cette technique, associée à un questionnement auto réflexif, constitue aussi une ai de au développement de la métacognition du sujet qui l'élabore par la réflexion d'auto-éval uation qu'elle suscite. Celleci a sans doute été renforcée dans notre étude par le concept central proposé. Une fois initiées l'acquisition du concept et la réflexion métacognitive, le dispositif de formation en alternance permet de travailler avec les participants le transfert de leurs compétences métacognitives dans leur pratique de formateurs en santé. Cette étude nous incite à proposer cette expérience de façon plus systématique auprès des nouvelles promotions de formateurs de professionnels de santé.

\section{Références}

1. Tardif J. Pour un enseignement stratégique, l'apport de la psychologie cognitive, M ontréal: Ed. Logiques, 1992.

2. Le Boterf $\mathrm{G}$. Compétence et navigation professionnelle, 3e éd., Paris: Editions d'O rganisation, 1999.

3. Raynal F, Rieunier A. Pédagogie : dictionnaire des concepts clés - Apprentissages, formation et psychologie cognitive, Paris: ESF, Pédagogies, 1997.

4. Baxter GP, Elder AD, G laser R. Knowledge-based cognition and performance assessment in the science classement. Educ Psychol 1996 ; 31 : 133-40.

5. Briscoe C, LaM aster SU. M eaningful learning in college bi ology through concept mapping. Am Biol Teach $1991 ; 53: 214-219$.

6. Schmid R, Telaro G. Concept mapping as an instructional strategy for high school biology. J Educ Res 1990; $84: 78-85$.

7. M oreira M , Concept maps as tools for teaching. J Coll Sci Teach $1979 ; 9: 283-286$.

8. N ovak JD, G owin D B. Learning how to learn, 4 e éd., N ew York : Cambridge U niversity Press, 1989.

9. Pinto AJ, Zeitz HJ. Concept mapping : a strategy for promoting meaningful learning in medical education. M ed Teach $1997 ; 19$ : 114-20.

\section{Remerciements}

$N$ ous remercions pour leur participation à cette expérience les participants en première année de la M aîtrise U niversi taire de Pédagogie des Sci ences de la santé, Bobigny, U niversité Paris 13, France: M esdames Béatrice D ecelle (Infirmière, Unité de Prévention et d'Education, H ôpital de D reux), M arie Josée Bernard D el orme (Sage Femme Indépendante en Suisse), Anne B onnerat (I nfirmière anesthésiste, SAM U du Centre H ospitalier de Guéret), et $M$ onsieur Jean- $F$ rançois $D$ eleris (Cadre infirmier, Institut de Formation en Soins I nfirmiers du Centre $\mathrm{H}$ ospitalier de M ontauban).

10. West D, Park J, Pomeroy R, Sandoval J. Concept mapping assessment in medical education : a comparison of two scoring systems. M ed Educ $2002 ; 36$ : 820-826.

11. M archand C, d'Ivernois J F, Assal J P, Slama G, H ivon R. An analysis, using concept mapping, of diabetic patient's knowledge, before and after patient education. M ed Teach $2002 ; 24: 90-99$.

12. Franca $S, M$ archand $C, C$ raplet $C$, Basdevant $A$, Ivernois ( $\left.d^{\prime}\right)$. Application of « concept mapping » in obese subjects : a pilot study in normo and underre porters. D iabetes M etab $2003 ; 29: 72-8$.

13. N adeau M-A. L'évaluation de programme, théorie et pratique Q uébec: Les presses de l'U niversité de laval, 2éd., 1990.

14. CarréP, M oisan A, Poi sson D. L'autoformation - pschopédagogie, ingénierie, sociologie. Paris: PU F, 1997.

15. Ausubel D. Educational psychology. A cognitive view, $2^{e}$ éd., $\mathrm{N}$ ew York : H olt, Rinehart and Winston, 1978.

16. Kinchin IM , H ay D B, Adams A. H ow a qualitative approach to concept map analysis can be used to aid learning by illustrating patterns of conceptual. Educational Research $2000 ; 42: 45-57$.

17. Stice C, Alavarez M . H ierarchical concept mapping in the early grades Childhood Education $1987 ; 64$ : 86-96. 